
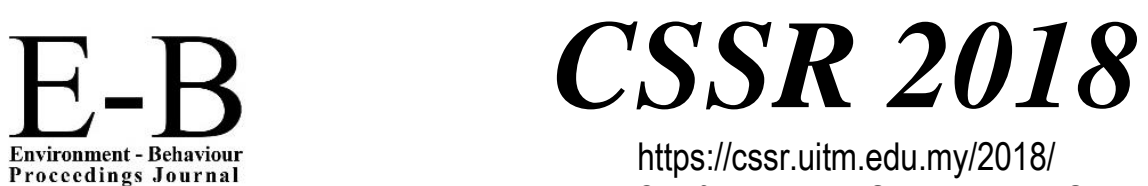

https://cssr.uitm.edu.my/2018/

5th International Conference on Science and Social Research Le Meridien Kota Kinabalu Hotel, 5 - 6 December 2018

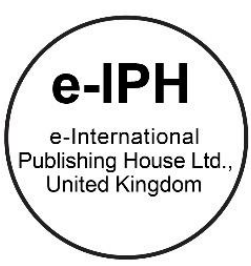

\title{
Conceptual Design of COD-E Humanoid Robots
}

\author{
Rusmadiah Anwar ${ }^{1}$, Verly Veto Vermol 2, Muhamad Fairus Kamaruzaman ${ }^{3}$ \\ ${ }^{1}$ Formgiving Design Research Group, Faculty of Art \& Design, Universiti Teknologi MARA, 40450 Shah Alam, Malaysia \\ rusma935@uitm.edu.my, verly@uitm.edu.my, fairuskamaruzaman@ieee.org \\ Tel: +603 55444001
}

\begin{abstract}
The conceptualizing process plays an important role in assisting designers' creativity in form and styling development. It contributes to representing the cultural elements before product transformation, which has a limited investigation. This research aims to identify the metaphorical form element that conveys the brain impaired as factors of selection and defining form development of the humanoid robot embodiment. Design Protocol Analysis obtains to into design linguistic interpretations and synthesizing design based on perceptual product experience. Findings have outlined the theory of metaphorical form element selection and identification that could represent brain impaired product in assisting humanoid robotic acceptance among autism.
\end{abstract}

Keywords: Design-Inspire; Humanoid Robot; Children with Autism; Design Protocol Analysis.

eISSN: 2398-4287@ 2020. The Authors. Published for AMER ABRA cE-Bsby e-International Publishing House, Ltd.. UK. This is an open access article under the CC BYNC-ND license (http://creativecommons.org/licenses/by-nc-nd/4.0). Peer-review under responsibility of AMER (Association of Malaysian Environment-Behaviour Researchers), ABRA (Association of Behavioural Researchers on Asians) and cE-Bs (Centre for Environment-Behaviour Studies), Faculty of Architecture, Planning \& Surveying, Universiti Teknologi MARA, Malaysia.

DOI: https://doi.org/10.21834/ebpj.v5iSl3.2534

\subsection{Introduction}

Assistive technology refers to a variety of devices for helping and assisting children with autism to work better within their daily context, education, environment and meet a higher quality of life and encompassing multiple resources which are providing different forms of practical supports that lead to behavioural and social benefits support for the end-users by reducing the negative impact of their disability conditions (Bauer, Elsaesser, \& Arthanat, 2011; Shih, 2011). Assistive technology offers role scope, such as playing and exploring by being creative in a highly controllable environment without any verbal demands. It is throughout assistive technology, cognitive can be integrated within components that enable scaffold learning (Kamaruzaman, Nor \& Azahari, 2016). Usually, children with autism process visual information easier than auditory information.

Meanwhile, design-inspire approaches can be critical when it comes to forming the development process (Anwar, Abidin, \& Hassan, 2015a; Corremans, 2011). As an artefact that reveals something insightful, conceptual metaphors are normally used in relating the connotation of separate entities to be conveyed and comprehended, thus allowing creativity during the form development of a product (Cila, Hekkert, \& Visch, 2014). When brain impaired specific information contributes to generating relevance to the product or design, it may deserve to be mentioned. However, it is often an issue of finding the right metaphor to design a humanoid robot. These days, Human-Robot Interaction (HRI) is a widespread activity that can be determined as an emerging research domain especially here in Malaysia. It is, however, before scientific investigation; incremental validity and research justification can be very challenging. In recent years, more and more robots are being used as assistive devices in the intervention as well as habilitation for

eISSN: 2398-4287C 2020. The Authors. Published for AMER ABRA cE-Bsby e-International Publishing House, Ltd., UK. This is an open access article under the CC BYNC-ND license (http://creativecommons.org/licenses/by-nc-nd/4.0/). Peer-review under responsibility of AMER (Association of Malaysian Environment-Behaviour Researchers), ABRA (Association of Behavioural Researchers on Asians) and cE-Bs (Centre for Environment-Behaviour Studies), Faculty of Architecture, Planning \& Surveying, Universiti Teknologi MARA, Malaysia.

DOI: https://doi.org/10.21834/ebpj.v5iSI3.2534 
children with a special need, and this also comprises children with autism (Stokes, 2000). Autism is a developmental disorder caused by abnormal development of the brain (Kamaruzaman, Noor, Hanapiah \& Azahari, 2016). The deficits in social and communication skills and behaviour stereotypes characterized the abnormal development. As autism is a lifelong challenge, intervention in the early years serves the purpose to cut the deficits of autism and to instil independent living abilities which could foster the children with autism quality of life (Kamaruzaman, Rahman, Abdullah, Anwar, 2013).

The robotic intervention has been recognized as one of the methods that are producing emboldening outcomes in the rehabilitation of children with autism. Robot-based solutions are desirable as they are inspiring, motivating, detailed, and repetitive. Reflecting Ho, MacDorman, and Pramono (2008), Mashahiro Mori, a Japanese robot was created to look identical to humans. The humanoid robots have the physical look similar to humans because they are divided into four sets of joints relating to the limb which is the legs and arms (Sian, Yokoi, Kajita, \& Tanie, 2003). Therefore, it can be simple when applying human gestures to the robotic form and produce a few motions and gestures. Gestures and postures are non-verbal communication because it is translated through human's physical gestures and not through oral communication. This research focuses on creating a variety of human gestures and postures that are applied to a robotic form.

\subsection{Humanoid Robot Design for Autism}

Based on the literature study, there is a lot of experiment including clinical research investigated between humanoid robots and children with autism engagement. Acknowledging this fact, metaphors are one of the mechanisms that let the well-known mental imagery of subjective experiences and judgments based on regular experiences which could be neutrally linked (Lakoff \& Johnson, 1999). These days, Human-Robot Interaction (HRI) is an emergent research domain here in Malaysia. Hence, it requires further study to prove its incremental validity and research justification that goes beyond scientific investigation. In recent years, more and more robots are being used as assistive devices in the intervention as well as habilitation for children with a special need, and this also comprises of children with autism. Autism is a developmental disorder caused by abnormal development of the brain. Deficits in social and communication skills and behaviour stereotypes characterized the abnormal disorder. As autism is a lifelong challenge, intervention in the early years serves the purpose to cut the deficits of autism and to instil independent living abilities which could foster the children with autism quality of life. The robotic intervention is one of the methods which are producing emboldening outcomes rehabilitate of children with autism. Table 1 has summarized a sample of robot-based solutions are desirable as they are inspiring, motivating, detailed, and repetitive.

Table 1. Humanoid robots tested to be interacted by child with autism

Description
2-feet-tall humanoid robot from Aldebaran Robotics.
Because of its success in the classroom, ASK (Autism Solutions
for Kids) Nao Program was launched.

(Source: NAO https://www.softbankrobotics.com/emea/en/nao; MILO https://robots4autism.com/milo; BUDDY https://buddytherobot.com/en/about-us/)

\subsection{A Complexity of Integrated Humanoid Robot Development in Design Process}

Product interactions could be achieved by their product perceptual experience (Warell, 2008). A framework of perceptual product experience, which outlined dimensions of 'presentation' and 'representation' bounded by a core mode categorized as 'experience." The representation dimension involved in the 'meaning-making' of product. 'Meaning' constitutes recognition, comprehension, and association with a particular product. In a product meaning association, metaphors recognize to express the characteristics and symbolic significance of the product. The framework of product experience (Desmet \& Hekkert, 2007) outlines the recognition. At some point, metaphors also benefitted the users as a medium of 'communication' to help them understand a product based on the inspiration which, captures from the appearance and attributes (Anwar, 2016). In replicating physical form close to human, it needs 
close human affection encounter whilst designer awareness throughout designing principal for the humanoid is mandatory. Design awareness in designing provides a designer with the ability to forecast product hindrances that may intrude on the users. It is also designed to outline limits dangers and the unfavourable results of coincidental or unintended activities. A protocol experiment has to put into practice in collecting important data of product performances and appearances that verifying users' experience that associates design with human affection. In return, the data can provide a bridge that potentially helps a designer in making sense of the user feedback drawn from using the product (Anwar, Abidin \& Hassan, 2016). Through experiences, user gauge cognitions of perception shaped by many factors. The process of perceiving understands and remembering explains the significant time constraint of knowledge building that involves input transfer within user-artefacts designer (see Fig. 1). User product experienced in a lab protocol environment amplifies critical both conscious and unconscious user patterns. Prevailing the answer to why it is necessary, what do they think and how do they behave from the task that sets in achieving the directed goal (Vermol, Anwar, Hassan, \& Abidin, 2015).

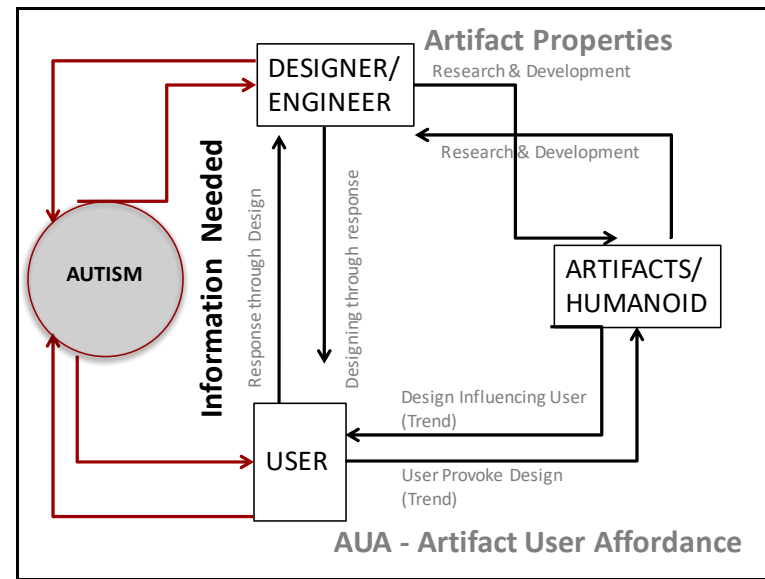

Fig. 1: AU-DA-Model. Reference for developing new concept of humanoid robot

(Source: Vermol, Anwar, Hassan \& Abidin, 2015)

\subsection{COD-E Humanoid Robot - Product Development}

Developing a conceptual humanoid robot requires certain issues to be solved. However, throughout the introductory of Critical Optimized Design through Emotion (COD-E) Humanoid Robot concept, innovative features of a shell design that protects the electronic parts become the critical influence that targeted to attracts interaction within the emotion of the user. As to plan out a new product in lines, as a supplement to existing market addition of humanoid via Bio-loid design of sizing and scaling (Fig. 2) has been repositioned to actual Bio-loid. Through design staging, the elaboration of the design process defined in 4 levels which is [1] ideate; [2] prototype screening; [3] fixing and fittings and; [4] digital characterizing.

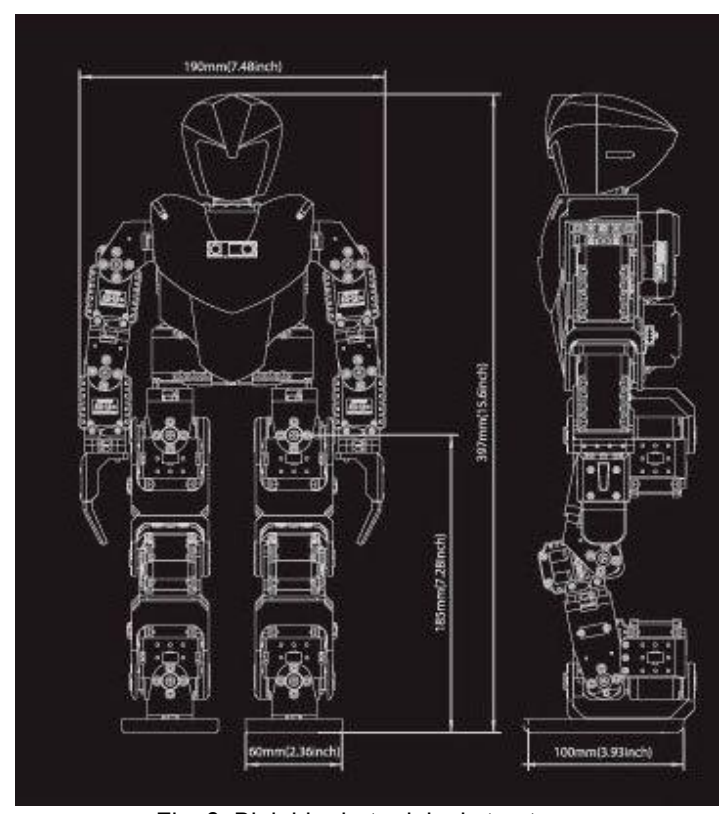

Fig. 2: Bioloid robot original structure

(Source: http://www.robotis.us/bioloid-1) 


\subsection{Ideate}

This research is to endow and developed a principle that will apply to any humanoid design for autism at any scale. The reasons for this hinge are to identify the relation between materials, shape, and the absolute dimension of design, its related accumulation, and the forces and deformations developed within it. The bigger an object gets, the more influential on form become structural issues. Ideating through a continuous, systematic search of new product sizing of attraction involves delineating sources of new ideas and methods based on principal design attributes. By taking into account the context in styling elaborations, the idea generated taking forms through design specification which, are fitted to compromise the partial aspect of Bio-loid original forms. Hence, the improvement made increases the humanoid appearance out of the original Bio-loid model. Fig. 3 shows a part of a design morphology based on the structural form of Bio-loid design. The investigations of the selection target area which revealed with poor ergonomic design, unsatisfactory, and quality problems. Ergonomics is a design-oriented discipline, ergonomists are just responsible for humanassociated design function and do not design the whole systems. But the COD-E design structure requires to satisfy multiple functional requirements including ergonomics, production process, time, and cost.

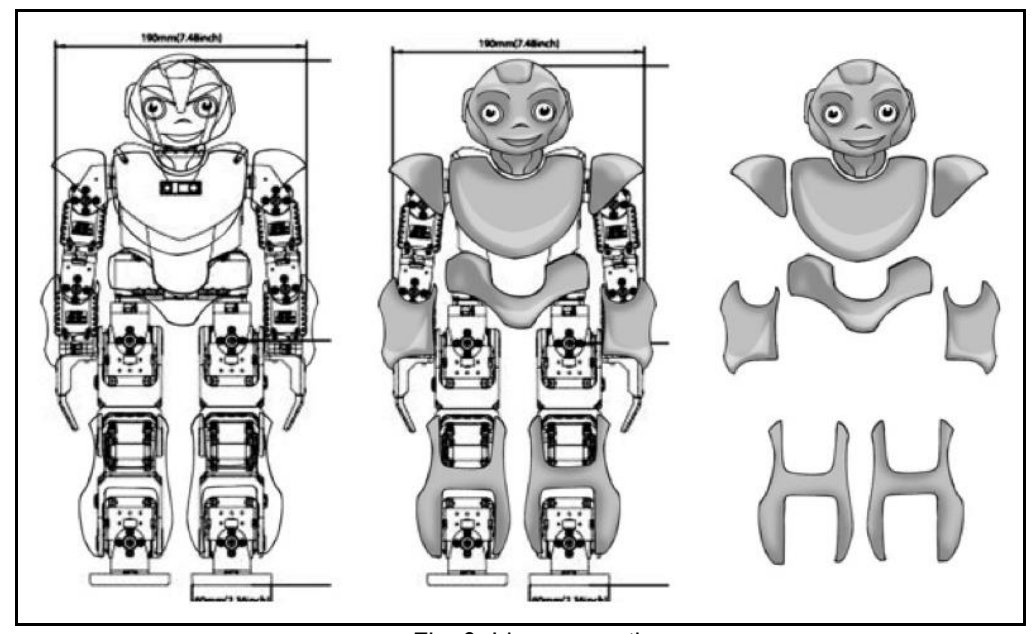

Fig. 3: Idea generation

\subsection{Prototype screening}

According to Ching (2008), Mashahiro Mori, a Japanese robot was created to look identical to humans. The humanoid robots have the physical look similar to humans by fragmenting four sets of joints relating to the limb which are the legs and arms (Yokoi, Kajita and Tanie, 2003). Therefore, it's easier to apply human gestures on a robotic form and produces a few motions and gestures. Gestures and postures are non-verbal communication. It is translated through human's physical gestures and not through oral communication. This study focuses on creating a robotic form through a variety of human gestures and postures. Through the generated idea, the design is then sculpted, fabricated, and fitted as a prototype sample to be deliberated and discussed. Through this process, screening is made following the aspect of the Bio-loid movement by taking account 'the state of pause, walk, and rest'. Through idea screening, fitting problems arises can be reduced to support Bio-loid progressive movement. Fig. 4 shows the idea of screening through the state and condition of Bio-loid.

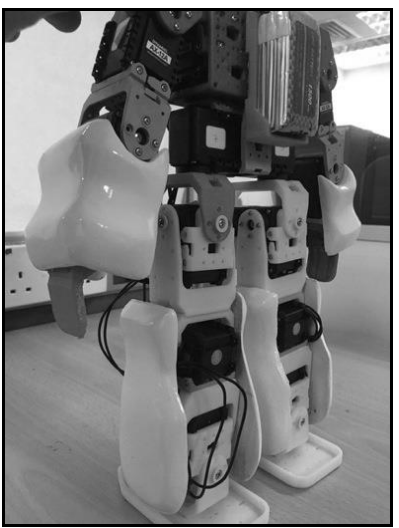

(a)

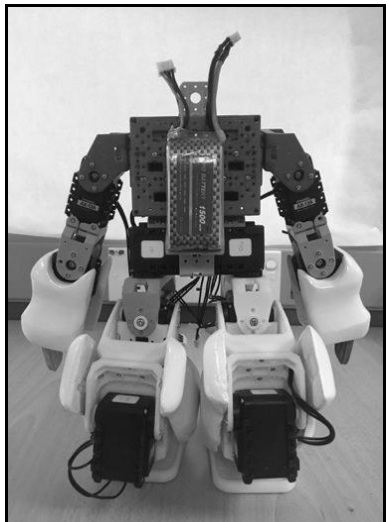

(b)

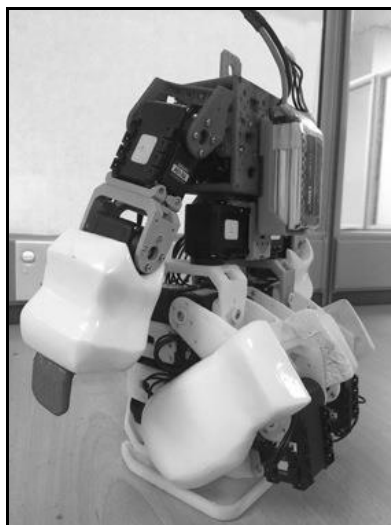

(c)

Fig. 4: (a) Side view through stand condition; (b) Front view through squat condition; (c) Side view through squat condition.

\subsection{Fixing and fitting}

Re-sizing the original Bio-loid anatomy is critical to support its movements and balance. During the fabricating stage, the stability set to the original Bio-Loid size and structure. It is, however, the location of brackets for the mini-motors remains on its original. 


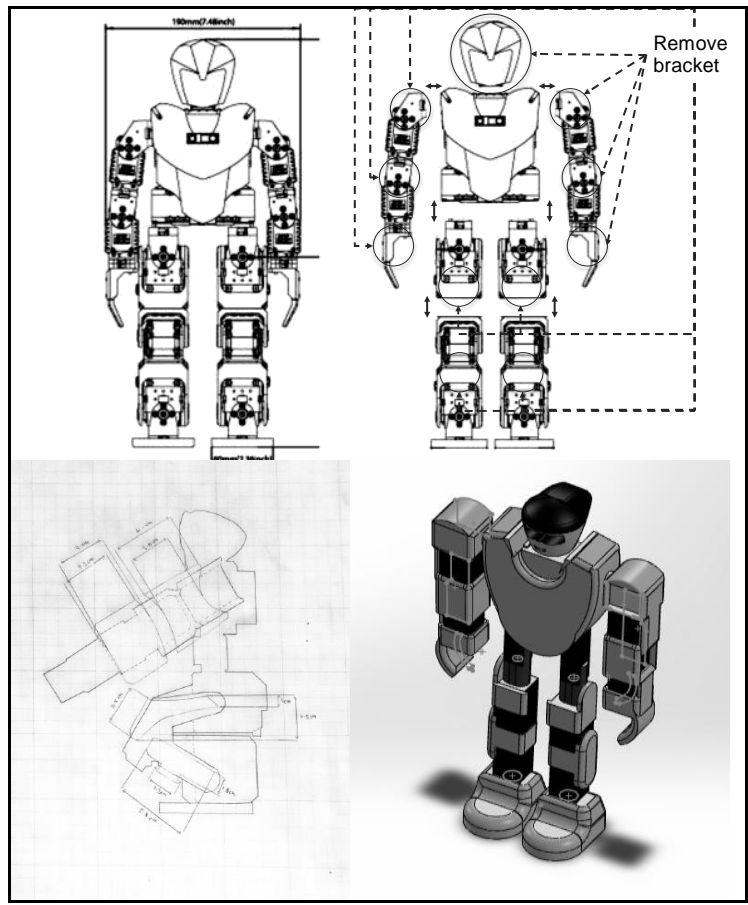

Fig. 5: Body concept development

\subsection{Digital characterizing}

Through digital concept visual development, characterization of each

humanoid-Bio-loid head design provides a series of COD-E Conceptual Design into consideration and discussion.

Selections of the ideation concept provide a closer step in bringing the final fabrication of the COD-E prototype into reality. The insight seeking and form element ordering during design episode has exposed the divergent-convergent of AFMA(Anwar, Abidin, \& Hassan, 2015b) thinking among designer generates a signi"cant visual surface shown in the form structure. Through the process, *ve conceptual designs of early COD-E were developed and to be re-codded. The result through circumstantial evidence adapted from AFMA approaches revealed that there is a consistent character arisen from the form-giving activities that relate to the human experience. Referring to the final concept of designers' impression design in Fig. 6 provides the overall improvement on appearance and sizing of Bio-loid new look. The success of form structure (body) has made possible the COD-E prototype design illustration complete as a whole (product gestalt) with any possible head design options.
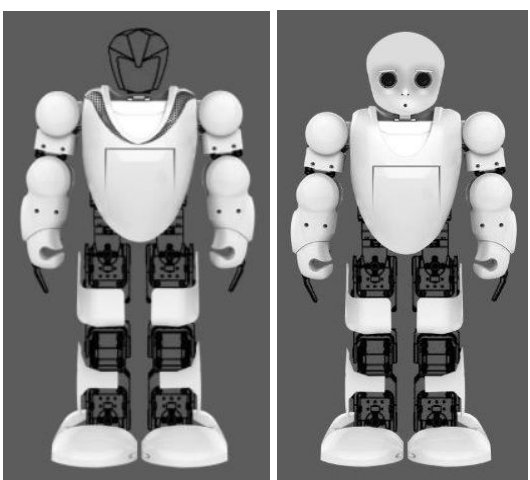

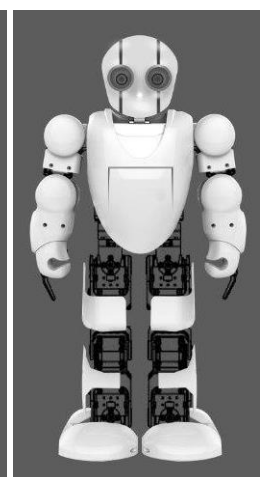

Fig. 6: Early concept of COD-E
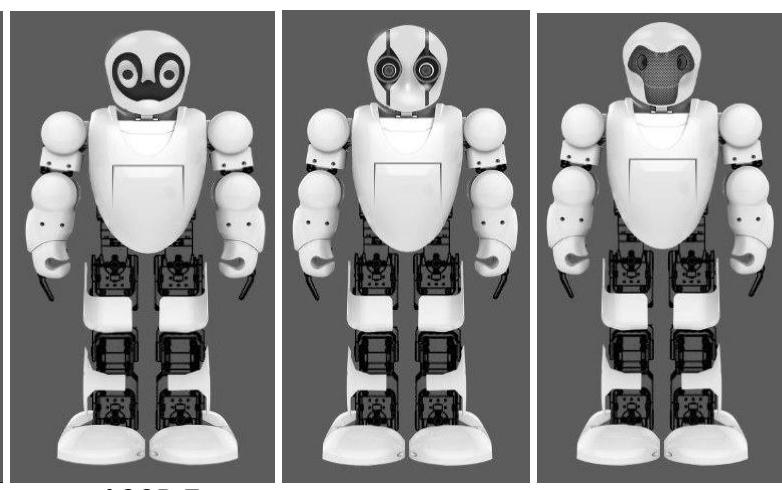

\subsection{Conclusions and future works}

The conceptual design process explained in this study features how

researchers observe primary users' experiences to re+ect on the experiment trial. Mediating through four humanoid prototypes of the same types and design with multiple facial expressions. As for future works, the researcher will be observing and summarizing human experiences through video recording within the contextual inquiry for finding a significant pattern. The signi ${ }^{\star}$ cance of this research underlines the design factors pattern draws out from the primary user representation that highlights pre-post performances. The results of the experiment generate the User-Designer consensus within the respondent's awareness. Makes invisible to the product designer enabling the researcher to look into gaps and articulate product performance according to the primary user perspective. COD-E design criteria demanded all materials and components that lead to long-term failure-free operation. Autism character on 
children debated as fundamental in creating better humanoid robot design and development. Thus the discussion focus only on structural design aspects. There are also technical issues that are non-structural. It is useful to review some of these issues as a way to understand design structural issues differences. Explained explicitly and implicitly on these studies to cater design development of a humanoid design. It can accrue through an attempt to improve the technical performance of COD-E design of any scale. For future works, the primary concern has usually been the improvement of the design, structure, or material itself. The consideration of facial expression will also need explorations towards matching the abilities of the autism needs with the COD-E structure design requirements.

\section{Acknowledgements}

The authors would like to acknowledge the Research Management Centre (RMC) of Universiti Teknologi MARA (UiTM) for the financial support under the Research Entity Initiative (REI) file number 600-IRMI/MYRA 5/3/REI (002/2018). Authors would also like to thank Formgiving Design Research Group team for the efforts and Faculty of Art and Design for all the project support.

\section{References}

Anwar, R. (2016). Characterizing a syntactic pattern of formgiving in design thinking process. Ph.D. Thesis. Shah Alam: Universiti Teknologi MARA.

Anwar, R., Abidin, S. Z., \& Hassan, O. H. (2015a). A pattern in formgiving design: Giving priority to a principle solution in industrial design situation. In Gen, M., Kim,K.J., Huang, X., Hiroshi, Y. (Eds.), Industrial Engineering, Management Science and Applications 2015 (pp. 331-340). Berlin Heidelberg: Springer.

Anwar, R., Abidin, S. Z., \& Hassan, O. H. (2015b). Function means analysis for ablution concept solution. The Turkish Online Journal of Educational Technology. Special Issue for INTE, 2015, 224-231.

Anwar, R., Abidin, S. Z. \& Hassan, O. H. (2016). In-vitro design protocol: Artificial situation strategy uses to comprehend designers thought. EDP Sciences: MATEC Web of Conferences. 52, 03002 .

Bauer, S. M., Elsaesser, L.-J., \& Arthanat, S. (2011). Assistive technology device classification based upon the World Health Organization's, International Classification of Functioning, Disability and Health (ICF). Disability and Rehabilitation: Assistive Technology, 6(3), 243-259.

Ching, C.H. (2008). Human Emotion of The Uncanny Valley: A GLS, MDS and ISOMAP Analysis of Robot Video Ratings, pp1, Indiana University, United States

Cila, N., Hekkert, P., \& Visch, V. (2014). Source selection in product metaphor generation: The effects of salience and relatedness. International Journal of Design, 8(1), $15-28$.

Corremans, J. A. (2011). Measuring the effectiveness of a design method to generate form alternatives: an experiment performed with freshmen student's product development. Journal of Engineering Design, 22(4), 259-274.

Desmet, P. M., \& Hekkert, P. (2007). Framework of product experience. International Journal of Design, 1(1), 57-66.

Ho, C.-C., MacDorman, K. F., \& Pramono, Z. D. (2008). Human emotion and the uncanny valley: A GLM, MDS, and ISOMAP analysis of robot video ratings. Proceedings of the 3rd ACM/IEEE International Conference on Human Robot Interaction. 169-176. paper \#10.1145/1349822.1349845.

Kamaruzaman, M. F., Noor, H. M., Hanapiah, F. A., \& Azahari, M. H. H. (2016). Efficacy of DTT by using touchscreen learning numeracy app for children with autism. Proceedings of the 2016 IEEE 8th International Conference on Engineering Education (ICEED). Langkawi, Malaysia, paper \#10.1109/ICEED.2016.7856071

Kamaruzaman, M. F., Nor, H. M., \& Azahari, M. H. H. (2016). Using touchscreen technology to support basic numeracy learning process for high functioning children with autism. The Turkish Online Journal of Educational Technology, 632-639.

Kamaruzaman, M. F., Rahman, S.H.A., Abdullah, K.Z., Anwar, R. (2013). Conceptual framework study of basic counting skills based dynamic visual architecture towards autistic children's development. Proceedings of the 2013 IEEE Business Engineering and Industrial Applications Colloquium (BEIAC). Langkawi, Malaysia, paper \#10.1109/BEIAC.2013.6560108

Lakoff, G. \& Johnson, M. (1999). Philosophy in the Flesh The Embodied Mind and Its Challenge to Western Thought. New York: Basic Book.

Shih, C.-H. (2011). Assisting people with developmental disabilities to improve computer pointing efficiency through multiple mice and automatic pointing assistive programs. Research in developmental disabilities, 32(5), 1736-1744.

Sian, N. E., Yokoi, K., Kajita, S., \& Tanie, K. (2003). Whole body teleoperation of a humanoid robot integrating operator's intention and robot's autonomy: an experimental verification. Proceedings of the 2003 IEEE/RSJ International Conference on Intelligent Robots and Systems (IROS 2003). Las Vegas, USA, paper \#10.1109/IROS.2003.1248881

Stokes, S. (2000). Assistive technology for children with autism. Published under a CESA 7 contract funded by the Wisconsin Department of Public Instruction.

Vermol, V., Anwar, R., Hassan, O., \& Abidin, S. (2015). Work in Progress: Mapping the development of quality sensuous response through Blind and Visually Impaired group (BVIG) touch interaction. Proceedings of the International Conference on Interactive Collaborative Learning (ICL), Florence, Italy, paper 25-29.

Warell, A. (2008). Modelling perceptual product experience-Towards a cohesive framework of presentation and representation in design. Proceedings of the 6th International Conference on Design \& Emotion 2008. Hong Kong, China, paper \#1-13 
Anwar, R., et.al., CSSR2018, 5th International Conference on Science and Social Research, Le Meridien Kota Kinabalu Hotel, 5 - 6 Dec 2018, E-BPJ, 5(SI3), Dec 2020 (pp.63-69)

Yokoi, E.S. Neo, Kajita, S. K. and Tanie K., (2003) "Whole Body Teleoperation of a Humanoid Robot Integrating Operator's Intention and Robot's Autonomy: An Experimental Verification," Japan, pp1652. 